\title{
Low Power MRI Techniques for Neurosurgical Planning and Post-surgical Assessment of Deep Brain Stimulators in Patients with Medically Refractory Parkinson's Disease or Dystonia
}

\author{
Subhendra N. Sarkar, ${ }^{1,2}$, Ron L. Alterman ${ }^{3}$, Efstathios Papavassiliou ${ }^{3}$, \\ Rafael Rojas ${ }^{4}$ \\ ${ }^{I}$ Department of Radiologic Technology \& Medical Imaging, New York City College of Technology, City \\ University of New York, Brooklyn, NY 11201 \\ ${ }^{2}$ Research Imaging Center, McLean Hospital, Harvard Medical School, Belmont, MA 02478; \\ ${ }^{3}$ Department of Surgery and ${ }^{4}$ Department of Radiology, Beth Israel Deaconess Medical Center, Harvard \\ Medical School, Boston, MA 02215.
}

\begin{abstract}
Medically refractory Parkinson's or dystonia conditions are often treated with deep brain stimulation (DBS) that are managed with the help of very restricted MRI due to safety concerns. The resulting MR images are often suboptimal but are still considered valuableto assess post-surgical complications as well as electrode placement accuracy. Absorbed radiofrequency power near the DBS electrodes during an MRI could produce tissue burns and,therefore, are subjected to strong conditional guidance from FDA and DBS manufacturers. We developed a comprehensive brain MRI protocol (including TI MPRAGE, FSET2, DTI and FSE IR with modified refocusing flip angles and stretched RF pulses) for 10 Parkinson's and 5 dystonia patients with implanted electrodes. This included low power $2 D$ and $3 D M R$ pulse sequences that preserved the expected tissue contrast, minimizedsignal artifacts and offered better tissue visualization near electrode edges. Low power images were judged as adequate for stereotactic planning in 12/15 patients while for the rest the neurosurgeons had to resort to additional landmarks. Radiologic diagnostic quality was also equivalent in tissue contrast and pathology detection as compared to high power routine MRI prior to electrode implantation. The white matter fiber tracks in DTI maps were virtually unchanged with minimal interference from the implanted leads.
\end{abstract}

Keywords: DBS: Deep Brain Stimulator/Stimulation, MRI: Magnetic Resonance Imaging, DTI: Diffusion Tensor Imaging, CT: Computed Tomography, RF power: Radiofrequency (induced) power (same as MRI heating), T1, MPRAGE, T2, FLAIR: various tissue contrast weighted MRI imaging methods

\section{Introduction}

Patients with advanced stages of Parkinson's diseaseor dystonia that are refractory to medical management may be quite effectively treated with deep brain stimulation (DBS). MRI has distinct advantages for mapping deep brain stimulation (DBS) lead position [1-3] and postoperative function[2,3]. However, local absorbed power during routine MRI image creation at the electrodes is several fold more than when electrode is absent in a routine head imaging, $[5,6]$ creating significant risks for such patients[7,8]. DBS manufacturers[9] have labeled their devices as magnetic resonance (MR) conditional by specifying upper limits for MRI generated heating to $0.1 \mathrm{~W} / \mathrm{kg}$ at 1.5 Tesla (T) and advised to use a local MRI excitation coil that does not apply power to chest electronic control system for a DBS patient (transmit/receive type head only MRI coil). The main reason for heating concern is due to the "critical length" of DBS leads (odd multiples of half wavelengths at 1.5 T), that could trigger very high and an unknown amount of local heating inside the brain[10] that varies with loop geometry and lead management from surgeon to surgeon.

Since a significant number of treatment-resistant patients are treated with such devices, a high-quality, low-power brain MRI could be valuable, although it is not currently available. For MRI of patients with DBS, some radiology groups have chosen to reduce the applied power by performing a limited MRI,[11,12] whereas others have used high-power MRI on DBS patients claiming that they have a low adverse incidence track record[13,14]. Note that a limited MRI may provide some protection against intra-or-extra cranial MRI burnsat the price of a significantly lower image quality. The safety and quality issues often discourages use of MRI for further MRI-guided neurosurgical interventionsand many surgeons opt to use CT instead. However, CT produces metal streaking artifacts with little cerebral tissue contrast or disease characterization compared to a high quality MRI. Our approach seem to provide adequate to high quality brain MRI sufficient for clinical diagnosis or additional neuro surgical planning.

We have developed a comprehensive brain MRI protocol utilizing both 2D and 3D MRI pulse sequences[15-17] with very low MRI radiofrequency power (RF power) ensuring a large RF safety margin while 
maintaining diagnostic image quality. Additionally we have identified which MRI sequences can localize DBS leadsbetter with respect to various deep brain stimulation targets and produce smallest metal signal artifacts allowing better tissue visualization at the electrode edges[18].

\section{Materials and Methods}

Using an IRB approved protocol 10 medically refractory Parkinson's disease patients (mean age range: 55-78, 4 females) and 5 dystonia patients (mean age range: 21-56, 3 females) were imaged for 2 sessions, first one for pre-DBS stereotactic planning at high RF power (3 W/kg max) at 1.5 or $3 \mathrm{~T}$ and the second session at 10 20 times lower RF power $(0.1 \mathrm{~W} / \mathrm{kg}$ max) at $1.5 \mathrm{~T}$ magnet (General Electric) for post-DBS electrode localization and screening for post-surgical complications. Various MRI sequences including T1 MPRAGE, FSET2, DTI and FSE IR sequences were used with prior sequence developmentsincluding modified MRI pulses for signal generation[13, 14]. White matter fiber tractography was performed by co-registeringdiffusion-weighted signal from fiber tracts on the underlying substrate images that were either low power 3D T1 or 2D T2 or 2D inversion recovery origin using the NordicICE Diffusion/DTI Module.

One neuro radiologist (RR) and one neurosurgeon (either EP or RA) compared the normal, high RF power images for pre-surgical planning and post surgical low power images with implanted metallic leads to assess potential post-surgical complications and lead location relative to desired target nuclei (Sub Thalamic Nuclei for Parkinson's and Globus Pallidal Nuclei or GPi for Dystonia). For assessing tissue contrast pre and post surgical images were compared using qualitative comparisons ofMRI appearance and tissue detectability for Globus Pallidus, Thalamus and Subthalamic Nucleus.The size of the signal void at each lead obliterating important structures were also noted since visualization of electrode/tissue interface is affected if the artifactual signal loss is substantial. However, the gray/white matter contrast is most important here as it localizes the electrode tip related to deep nuclei of interest.

\section{Results and Discussion}

There were no patient-related complications or device malfunctions during or after MRI. Low-power images on implant patients demonstrated tissue contrast comparable to images at high-power from non-implant sessions and considered adequate for radiologic interpretations.

All three readers concluded that low and high power images did not differ substantially in terms of tissue contrast and the extent of signal void due to metallic artifact from DBS tips was acceptable for diagnostic assessment or further surgical planning. However, low power images were reliable for stereotactic planning for $12 / 15$ patients; while for the rest the neurosurgeons had to use additional landmarks for target verification due to suboptimal MR image quality. In all surgical sessions, in addition to using the MR images, real time patient feedbacks were also used during electrode advancement inside the brain parenchyma to confirm or adjust the electrode depth to ensure improved motor functionality and target response for best treatment outcomes.

Spin echo based low RF power MRI sequences provided acceptable image quality with minimum image blooming (enlargement, Fig 1B, 1C) while MPRAGE, a gradient echo based T1 sequence produced maximum signal void at the stimulator leads (Fig 1A). The mean lead diameters visualized on images including the artifactual signal voids were $2.1 \mathrm{~mm}$ for 2D, $2.2 \mathrm{~mm}$ for $3 \mathrm{D}$ spin echo based sequences (T1, T2 and FLAIR) and 3.6-4.0 $\mathrm{mm}$ for 3D MPRAGE sequencewhen averaged over the entire patient group while the true diameters for the electrode tips was only about $1.3 \mathrm{~mm}$.

The gray and white mattertissue contrasts for two dimensional low power T2 (Figure 1C) and Inversion Recovery MR images (Figure 3) were adequate for stereotactic targeting while the 3D T1 MPRAGE or spin echo T1 images (Figure 1A and 1B) could only be used to establish an indirect tissue mapping relationship using a typical stereotactic atlas.

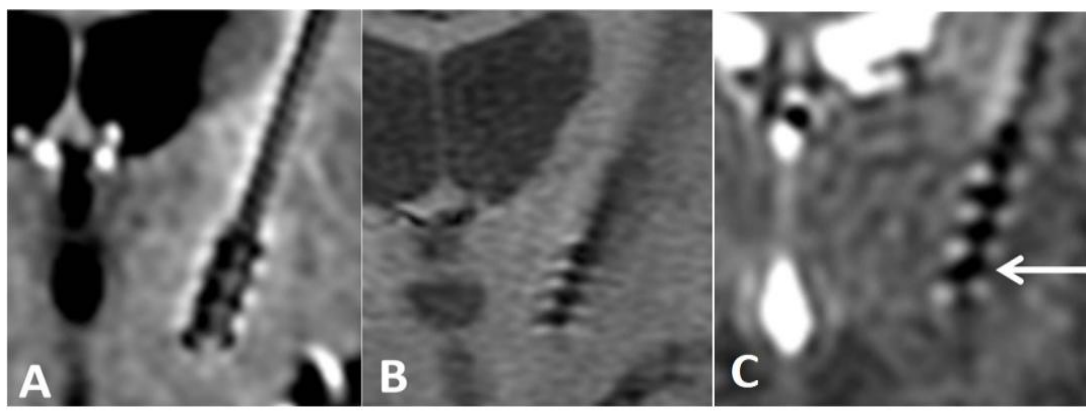

Figure 1. Typical coronal MR images, (A) a section from 3D MPRAGE; measured contact diameter (shown in panel $\mathrm{C}$ by straight arrows) $=3.6 \mathrm{~mm}$, more than that from $(\mathrm{B})$ a section from 2D Spin Echo T1 (2.1 mm) or $(\mathrm{C})$ Coronal 3D Spin Echo T2 image showing $2.2 \mathrm{~mm}$ tip diameter. 
Note that the detection and management of an infected DBS tip was successfully done using very low power 3D MRI FLAIR images (Figure 2). Routine 2D FLAIR MRI usually generates an unacceptable MRI heating and was not used.

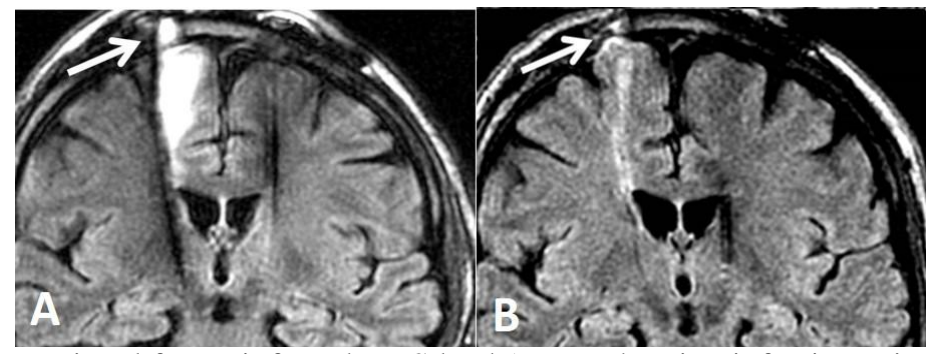

Figure 2. 3D FLAIR MRI signal for an infected DBS lead (arrow showing infection prior to removal, (A) and 2 months later, after implant removal and treatment by antibiotics (B). The contralateral lead was not infected.

In Figure 3 high power pre-surgical inversion recovery MRI image is shown (3A) followed by post-left DBS implantationimage performed same day at low power (3B) and image from post-right DBS implantation (3C). Readers agreed that the high power $(3 \mathrm{~A})$ and low power $(3 \mathrm{~B}, 3 \mathrm{C})$ images have almost identical tissue contrast.

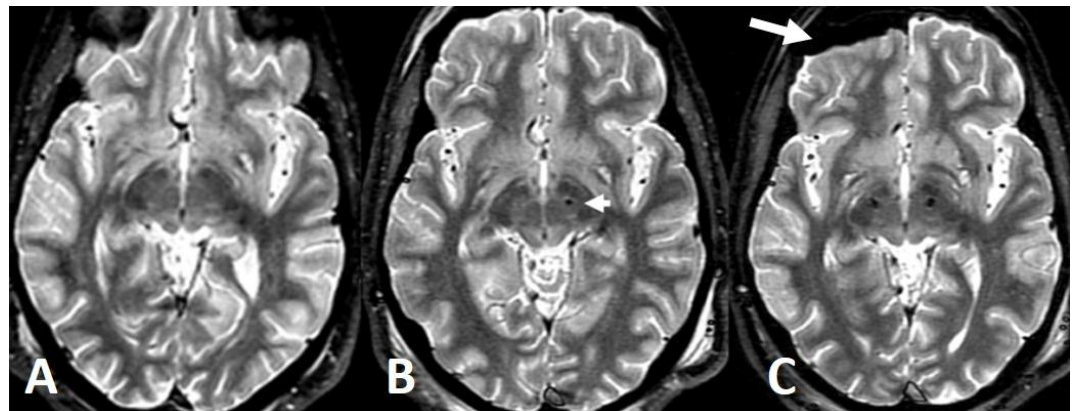

Figure 3. Typical Fast Spin Echo Inversion Recovery images: (A) at power level of $1.5 \mathrm{~W} / \mathrm{kg}$ for pre-surgical planning; (B) at a power of $0.1 \mathrm{~W} / \mathrm{kg}$ after $1^{\text {st }} \mathrm{DBS}$ implantation to assess the surgical accuracy of the $1^{\text {st }}$ lead and also for surgical planning for $2^{\text {nd }} \mathrm{DBS}$ and $(\mathrm{C})$ at a power level of $0.1 \mathrm{~W} / \mathrm{kg}$ after the $2^{\text {nd }}$ lead placement for assessing surgical accuracy and complication.

In Figure 4, the fractional anisotropies from DTI maps at basal ganglia level were independently measured by two readers and seemed to remain unchanged (for all 3 track overlays: MPRAGE or T2 or inversion recovery methods. The last two imaging methods delineated deep nuclear boundaries with significantly better G/W contrast. The high-power (pre-DBS) and low-power (post-DBS) DTI fiber tracts were qualitatively similar with minimal interference or artifactual distortion from the implanted leads. For deep brain stimulation treatments the knowledge about the pre-and-post surgical white matter connectivity could be very helpful. Low power MRI allows assessment of white matter integrity in patients with DBS and may be used to monitor the connectivity changes, alter stimulation parameters and minimize adverse side effects with DBS treatment.

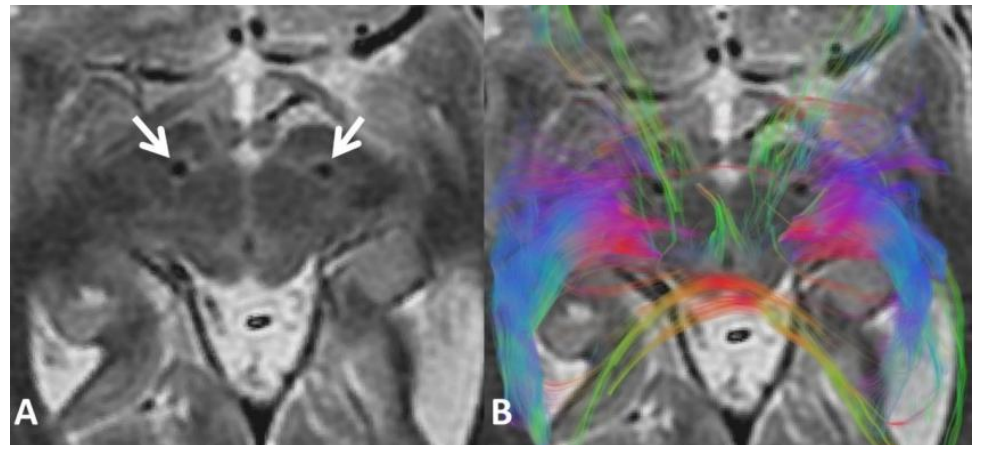

Figure 4. (A) Post-surgical, low power inversion recovery image, arrows point to two DBS electrode tips at the level of subthalamic nuclei and (B) white matter fiber tracks using diffusion tractography superimposed on the same image as in (A). The fiber tracks seem to be unaffected by the presence of electrodes. 
Due to the unavailability of low-power MR methods (at FDA approved level) many patients with implanted deep brain stimulators (DBS) do not benefit from all clinically useful MR sequences. The current practice of using atlas-based, indirect targeting of deep nuclei is imperfect. Recent reports claim diffusion tractography improves targeting accuracy also show significant variation when MRI is used to co-localize cingulate gyrus targets in depression(1) or thalamic targets for tremor(2). Such issues may be resolved if advanced techniques like DTI can be routinely applied at FDA approved low-SAR level for patients with indwelling electrodes. We propose localizing DBS leads by T2 and FSEIR images that are more useful for delineating deep nuclear boundaries than conventional T1 MPRAGE followed by overlay of white matter fibers, all at low-power using the proposed low-power sequences. In the near future it may be possible to monitor such patients neurological function by low power functional MRI as demonstrated by functional MRI mapping forParkinson's patients with implanted DBS electrodes[4, 20].

\section{Study Limitations}

Low RF power MRIthat we have developed can minimize the potential for RF heating although it cannot completely eliminate a nominal amount of heating[15-17].There is controversy about the iron composition and MRI contrast of deep brain nuclei as studied on a small patient population [19] and by other researchers. Producing adequate MRI tissue contrast for various neurosurgical targets is substantially challenging for low or high power MRI and robust MRI sequences need to be developed. Preservation of tissue contrast and identifying post-surgical lead locations as shown here should be applied to larger patient cohorts and experience from multiple neurosurgical practice sites are needed to appreciate the utility of low power MRI.

\section{Conclusions}

Diagnostic quality brain MRI can be performed with optimized, 2D or 3D sequences in patients with neuro implants within restrictive RF safety guidelines by significantly reducing tissue heating risks. Image quality and relative tissue contrasts comparable to clinical standards are achieved adjacent to DBS leads as well as in distal brain regions using low RF power spin echo based 2D and 3D sequences. The smallest artifactual blooming of stimulator leads happens in 2D and 3D FSE sequences while the highest signal void appears in the 3D MPRAGE sequence, being a gradient echo method.Low power (post-DBS, 1.5T) DTI fiber tracts were adequately visualized with minimal distortion or interference from the implanted leadssuggestive of postsurgical white matter fiber integrity in this patient population and may be used for future lead implantations or monitor existingDBS treatment.

\section{Acknowledgements}

The authors thank David Hackney MD, Michael Fox MD and Rafeeque Bhadelia MD for valuable discussions on various aspects of DBS related neuro imaging.

[1]. Wodarg F, Herzog J, Reese Ret al.Stimulation site within the MRI-defined STN predicts postoperative motor outcome. Mov Disord2012;27:874-879

[2]. Zrinzo L et al. Clinical Safety of Brain Magnetic Resonance Imaging with Implanted Deep Brain Stimulation Hardware: Large Case Series and Review of the Literature. World Neurosurg 2011;76:164-172.

[3]. Bhatia S, Oh M, Whiting T et al. Surgical complications of deep brain stimulation. A longitudinal single surgeon, single institution study. Stereotact Funct Neurosurg 2008; 86:367-72 and Stereotact Funct Neurosurg 2012;90:225

[4]. Boertien T, Zrinzo L, Kahan J et al.Functional imaging of subthalamic nucleus deep brainstimulation in Parkinson's disease. Mov Disord2011;26:1835-1843.

[5]. Bottomley P, Roemer R. Homogeneous tissue model estimates of SAR deposition inhuman NMR studies. Local elevations predicted in surface coil decoupling. Ann NY Acad Sci1992; 649:144-159.

[6]. Collins CM, Liu W, Wang Jet al.Temperature and SAR calculations for a human headwithin volume and surface coils at 64 and 300 MHz. J Magn Reson Imaging2004;19:650-656.

[7]. Sharan A, Rezai AR, Nyenhuis JA et al.MR safety in patients with implanted deepbrain stimulation systems (DBS). Acta Neurochir Suppl2003;87:141-145.

[8]. Rezai AR, Finelli D, Nyenhuis JA et al.Neurostimulation systems for deep brainstimulation: in vitro evaluation of magnetic resonance imaging-related heating at 1.5 Tesla. J Magn Reson Imaging2002;15:241-250

[9]. Medtronic. MRI Guidelines for Medtronic Deep Brain Stimulation Systems. Minneapolis,MN: Medtronic Inc.; 2007. Available at: http://professional.medtronic.com/wcm/groups/mdtcom_sg/@mdt/@neuro/documents/documents/dbs-2007-mri.pdf.

[10]. Kainz W. MR Heating Tests of MR Critical Implants. Editorial, J Magn Reson Imaging2007;26:450-51.

[11]. Kovacs N, Nagy F, Kover F et al.Implanted deep brain stimulator and 1.0-Tesla magneticresonance imaging. J Magn Reson Imaging2006;24:1409-1412.

[12]. Huston OO, Watson RE, Bernstein MA et al.Intraoperative magnetic resonance imagingfindings during deep brain stimulation surgery. J Neurosurg2011;115:852-857.

[13]. Larson PS, Richardson RM, Starr PA et al. Magnetic resonance imaging of implanteddeep brain stimulators: experience in a large series. Stereotact Funct Neurosurg2008;86:92-100.

[14]. Starr PA, Christine CW, Theodosopoulos PV, et al. Implantation of deep brain stimulators into the subthalamic nucleus: technical approach and magnetic resonance imaging-verified lead locations. J Neurosurg 2002;97: 370-87.

[15]. Sarkar S, Alsop DC, Madhuranthakam AJ et al.Brain MR imaging at ultra-low radiofrequency power. Radiology2011;259:550-557. 
[16]. Sarkar SN, Papavassiliou E, Rojas R et al. Low-Power Inversion Recovery MRI Preserves Brain Tissue Contrast for Patients with Parkinson Disease with Deep Brain Stimulators. AJNR Am J Neuroradiol 2014;35:1325-9.

[17]. Sarkar SN, Papavassiliou E, Hackney DB et al. Three-dimensional brain MRI for DBS patients within ultra-low radiofrequency power limits. Movement Disorders Journal 2014;29: 546-49.

[18]. Sarkar SN, Sarkar PR, Papavassiliou E et al. Utilizing Fast Spin Echo MRI to Reduce Image Artifacts and Improve Implant/Tissue Interface Detection in Refractory Parkinson's Patients with Deep Brain Stimulators. Parkinson's Disease Journal 2014; 508576.

[19]. Sarkar SN, Sarkar PR and Papavassiliou E. Subthalamic Nuclear Tissue Contrast in Inversion Recovery MRI Decreases with Age in Medically Refractory Parkinson's Disease. J of Neuroimaging 2015; 25: 303-6.

[20]. Sarkar SN, Sedora-Roman NI, Fox MD et al. Resting state functional connectivity in Parkinson's patients with implanted deep brain stimulation electrodes. Abstract, $101^{\text {st }}$ Meeting, Radiologic Society of North America, Nov 29-Dec 4, 2015, Chicago, IL. 